\title{
BMJ Open Interventions to encourage smoke-free homes in remote indigenous Australian communities: a study protocol to evaluate the effects of a community- inspired awareness-raising and motivational enhancement strategy
}

\author{
Alan R Clough, ${ }^{1}$ Kristy Grant, ${ }^{1}$ Jan Robertson, ${ }^{2}$ Matthew Wrigley, ${ }^{3}$ Nina Nichols, ${ }^{4}$ \\ Tracey Fitzgibbon ${ }^{5}$
}

To cite: Clough AR, Grant K Robertson J, et al. Interventions to encourage smoke-free homes in remote indigenous Australian communities: a study protocol to evaluate the effects of a communityinspired awareness-raising and motivational enhancement strategy. BMJ Open 2018;8:e018955. doi:10.1136/ bmjopen-2017-018955

- Prepublication history for this paper is available online. To view these files, please visit the journal online (http://dx.doi. org/10.1136/bmjopen-2017018955).

Received 8 August 2017 Revised 7 February 2018 Accepted 13 February 2018

\section{Check for updates}

${ }^{1}$ Tropical Medicine and Rehabilitation Sciences, James Cook University, Cairns, Queensland, Australia

${ }^{2}$ College of Healthcare Sciences, James Cook University, Cairns, Queensland, Australia

${ }^{3}$ Aboriginal Resource

Development Services (ARDS), Darwin, Australia

${ }^{4}$ Apunipima Cape York Health Council, Bungalow, Queensland, Australia

${ }^{5}$ Aboriginal Land Progress Association (ALPA), Darwin, Australia

Correspondence to Professor Alan R Clough; alan.clough@jcu.edu.au

\section{ABSTRACT}

Introduction Rates of secondhand smoke exposure are currently significantly higher among remote indigenous communities in the top end of Australia. By implementing a 'smoke-free home' rule, secondhand smoke exposure can be reduced. Smoke-free homes encourage quit attempts and improve the health of children. The prevalence of indigenous smoking rates in remote, discrete communities in Australia is elevated compared with their nonindigenous counterparts. The primary aim of this project is to examine the feasibility of conducting a health-driven intervention to encourage community members to make their homes a smoke-free zone.

Methods and analysis This study uses mixed-methods exploratory evaluation design to obtain data from key informants and community householders to assess their willingness to implement a 'smoke-free' rule in their homes. Initial focus groups will provide guidance on intervention content and deliver evaluation procedures and community requirements. A rapid survey will be conducted to ascertain interest from community members in having the project team visit to discuss study objectives further and to have a particle meter (with consent) placed in the house. Focus groups recordings will be transcribed and analysed thematically. Rapid surveys will be analysed using frequency distributions and tabulations of responses. Ethics and dissemination The National Health and Medical Research Council guidelines on ethical research approaches to indigenous studies will be adhered to. The James Cook University Human Research Ethics Committee has provided ethics approval.

\section{INTRODUCTION}

In the world's indigenous populations, there are no published evaluations of interventions to reduce secondhand smoke exposure (SHSe) in indigenous homes. However, there is good evidence for the general population that, at every opportunity, SHSe should be
Strengths and limitations of this study

- Strong coalition of community-based partners.

- Communities have shown willingness to participate.

- Due to limited resources, only a small number of communities can be included.

- No control communities and no random selection of either communities or households.

minimised as it increases the risk of allergies, neural tube defects, sudden infant death syndrome and exacerbates asthma in children $^{1-3}$ while also increasing the risks for lung cancer, ischaemic heart disease and respiratory disease in adults. ${ }^{34}$ An estimated 600000 (around 1.0\%) of deaths worldwide are attributed to SHSe, $28 \%$ of which are deaths in children. ${ }^{1}$ Moreover, there is good evidence that a smoke-free home: (1) reduces SHSe for resident non-smokers (particularly for children $) ;^{5}$ (2) increases the number of quit attempts among active smokers in households ${ }^{56}$ and (3) deters resident adolescents from becoming established smokers. 178 Studies in indigenous populations examining the use of incentives, competitions and sponsorship, combined with other strategies to reduce smoking, have been conducted with indigenous pregnant women, on worksites and among students. ${ }^{9-13}$ The potential advantages of using incentives specifically to encourage smoke-free homes have not been examined. Therefore, little is known about whether and what kind of incentives may be relevant and acceptable in indigenous populations. Finally, for the general population, several studies have measured SHSe and used 
the information to mobilise household-level change. ${ }^{14-20}$ However, no such studies in indigenous populations have been published.

This formative research of tobacco SHSe will be conducted in selected Aboriginal and Torres Strait Islander (indigenous) communities in Northern Australia. A coalition of community-based partners with a long history of engagement with their constituent communities in this region have designed an innovative suite of strategies. Community-wide awareness-raising programmes about the health risks of SHSe have been developed based on long-standing, cross-cultural 'Discovery Education' approaches, which is based on open communication with community members. 'Discovery Education' will impart information about the benefits of smoke-free homes in particular, and about managing smoke-free spaces generally, after its translation into locally accessible language and conceptual constructs. Measures of respirable particulate levels will be taken in households and the meaning and implications of these explained in terms of SHSe. At the same time, the partners propose to design and administer culturally relevant and acceptable incentives for householders to reinforce existing smoke-free home strategies. The evaluation of this initiative described in this protocol will take a collaborative approach and will rigorously assess the outcomes achieved and the processes used by this coalition of community service providers, indigenous community leaders and community advocates.

There is a high need for evidence regarding any interventions that could work because smoking rates are elevated in indigenous populations. In North America and Oceania, where indigenous peoples were colonised by peoples from Western Europe, consistent, contemporary disparities in smoking rates exist between the local indigenous populations and the general populations of countries in these regions and these run parallel to the known disparities in tobacco-related morbidity and mortality. ${ }^{21}{ }^{22}$ In Native Americans, current daily smoking rates of $21.9 \%$ are 1.3 times higher than for other American. ${ }^{4}$ In Canada, smoking rates among Métis, First Nations and Inuit populations of $36.8 \%, 40.1 \%$ and $49 \%$, respectively, are from 2.5 to 3.3 times higher than for other Canadians. ${ }^{23}{ }^{24}$ Daily smoking rates among New Zealand Maori of $38.6 \%$, are 2.4 times those for non-indigenous New Zealanders. ${ }^{25}$ Current daily smoking rates of $38.9 \%$ among Aboriginal and Torres Strait Islander (indigenous) Australians are three times higher than for non-indigenous Australians ${ }^{26} 27$ with tobacco use still the leading preventable cause of the disproportionately high chronic disease burden compared with other Australians. ${ }^{26} 2829$

It is well documented in Australia that these disparities in smoking rates and disease burden widen with increased remoteness and isolation. ${ }^{26}{ }^{30}$ Adding to the burden, overcrowding of housing increases with remoteness and isolation in the same manner as smoking rates. ${ }^{29} 31$ From $60 \%$ to $71 \%$ of all indigenous Australians live in households with daily smokers ${ }^{131}$ and overcrowding of houses in indigenous Australian communities reinforces high-smoking rates and increased SHSe. ${ }^{32}$ The potential impacts of SHSe are thereby compounded providing further justification for intervention strategies that are robust and community driven combined with rigorous evaluation studies that can clearly demonstrate effectiveness.

Our pilot data indicate that in response to this widespread tobacco use, remote community residents wish to reduce smoking in their homes to protect the health of their children and to support quit attempts by smokers in their families. ${ }^{6-8}{ }^{33-35}$ In four participating remote indigenous communities in Northern Australia, this study will: (1) apply the 'Discovery Education' approach to awareness raising about the health risks of SHSe and examine its utility and transferability; (2) design locally relevant incentives to encourage the creation of smoke-free homes and assess the acceptability of the incentives; (3) measure SHSe in participants' homes and investigate the use of these measures for mobilising change at the household level while, at the same time and (4) monitoring tobacco sales through local community stores. The protocol for this evaluation study is designed to support these community-inspired efforts, in order to refine the community partners intervention strategies, to consider their transferability and to test elements of a robust methodology to implement the intervention elsewhere and to evaluate its effectiveness with valid outcome measures in a larger trial.

\section{METHODS}

\section{Study setting: 'very remote' indigenous communities}

The highest smoking rates known for indigenous Australians have been found in the 'very remote' communities where this study is located (see figure 1). Smoking rates of $61.7 \%$ of adults have been documented in far North Queensland ${ }^{30}$ and between $71 \%$ and $82 \%$ in the 'Top End' of the Northern Territory (NT), ${ }^{31} 3236$ substantially higher than $38.9 \%$ for indigenous Australians overall. ${ }^{26} 27$ Remarkably, these rates have not changed since the 1980s when $76.5 \%$ of Aboriginal adults surveyed in this part of the NT were smokers. ${ }^{37}$ Data from national surveys consistently reflect this lack of change in smoking rates in remote localities across Australia, ${ }^{35}$ 38-42 contrasting with a reduction in smoking rates in the past decade among indigenous Australians overall, from $51 \%$ to $44 \%{ }^{31}$

In official statistics in Australia, the category of 'very remote' indigenous communities like those in this study, typically describes very small clusters of people and dwellings. ${ }^{43}$ They range in size from isolated 'outstations' housing just a few families, to larger settlements established and maintained during the early 20th century as Christian missions or Government stations with today around 3500 people living in the largest of these. ${ }^{44}$ While English is widely spoken in most communities, local languages are also retained and used as the first language in many places. Their populations are among the 


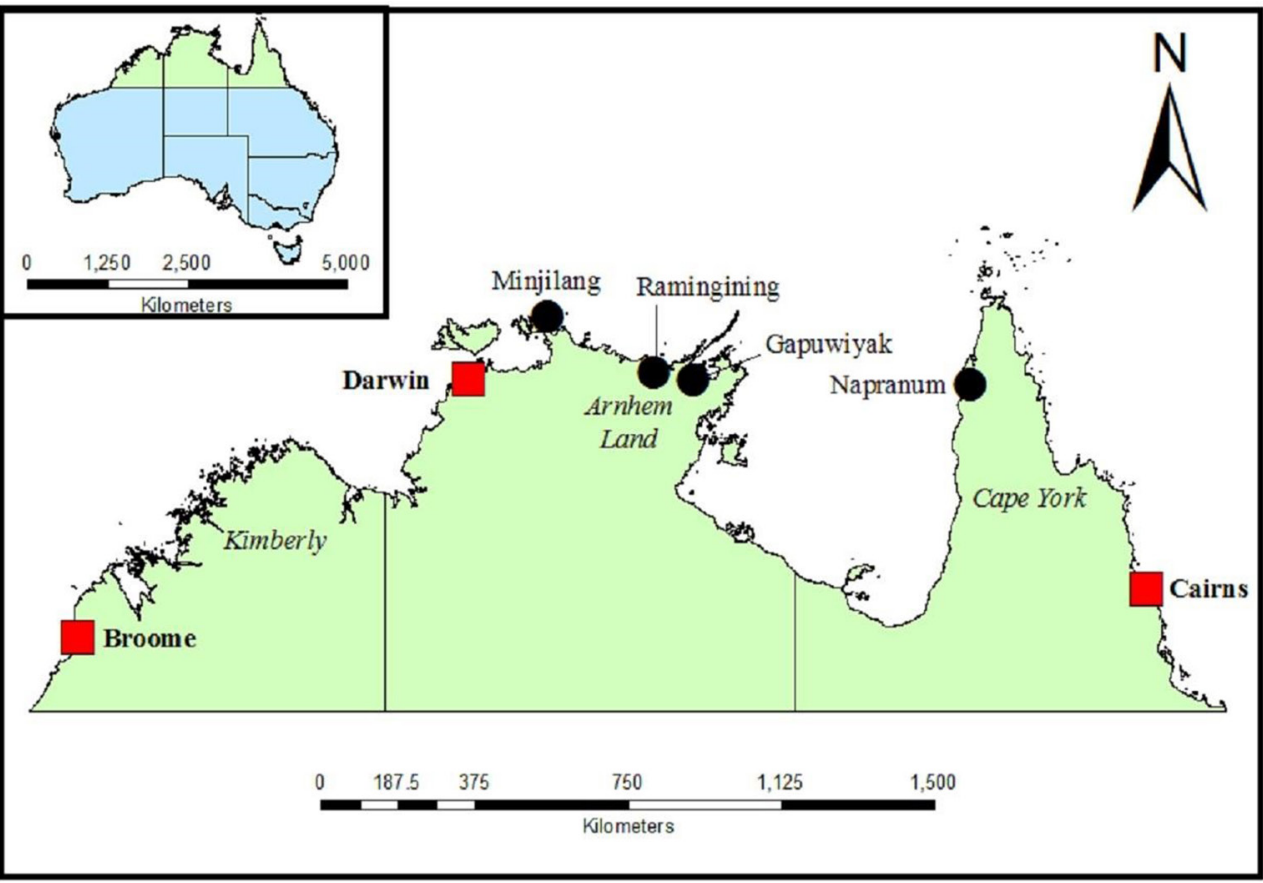

Figure 1 Map created with ESRI ArcGIS using ABS Data. ${ }^{73}$ Red, major cites; black, indigenous communities.

more severely disadvantaged and vulnerable groups in Australia; physically and socially isolated from the mainstream economy, and bearing a disproportionate burden of preventable chronic conditions, especially those linked with substance misuse. ${ }^{45} 46$ The total 'very remote' indigenous Australian population is approximately 92000 , equivalent to $13.7 \%$ of Australia's total indigenous population but constituting a very small proportion $(0.4 \%)$ of all Australians. ${ }^{44}$

The people living in such remote localities, including those in this study, have done so for much of their lives. Unique traditional cultural practices are maintained with relationships between individuals tightly circumscribed, regulated by cultural rules and expectations across long-established family and clan groups. ${ }^{47}$ The available housing typically accommodates more people than its construction is designed to support in these communities $^{42}$ and there are few opportunities locally for mainstream employment. Cultural and family obligations mean that all resources are shared ${ }^{48}$ particularly desirable ones like tobacco, along with the money to purchase it. ${ }^{49}$ Tobacco is routinely shared among users. ${ }^{50}$

\section{Study partners}

This partnership has evolved over two decades. Apunipima Cape York Health Council (Apunipima) is the largest community controlled health organisation in Queensland. ${ }^{51}$ It provides health services to the indigenous residents of Cape York communities using social and emotional well-being approaches. ${ }^{52}$ In the NT, the Aboriginal Resource and Development Services (ARDS) Aboriginal Corporation is a cross-cultural communication and media specialist organisation which delivers communication services designed to bridge the knowledge gap between mainstream services and community people. ARDS has particular cross-cultural and linguistic expertise in Arnhem Land Yolngu matha speaking populations in the NT's 'Top End'. ${ }^{53}$ The Arnhem Land Progress Association Aboriginal Corporation (ALPA) provides retail services in community stores in remote communities across both far North Queensland and in the NT, including in all four of the study communities. As part of their product lines, ALPA makes tobacco products available locally. ${ }^{54-56}$ Although tobacco products can be purchased in larger regional centres, residents in the study communities generally prefer to purchase tobacco at the local ALPA store. ${ }^{32}$

\section{Participating study communities}

Four communities have provided their formal permission to participate in the collaborative intervention effort and in this evaluation study. Three are in the 'Top End' of the NT while one is in far North Queensland (figure 1). Napranum is situated on the Western side of Cape York in far North Queensland near the mining town of Weipa and $625 \mathrm{~km}$ from Cairns, the main regional centre. At the 2016 census, Napranum, with the least crowded housing, was comprised 228 households for its 907 indigenous residents, ${ }^{57}$ around 4.0 persons per household. Minjilang, in Western Arnhem Land (NT), is located $235 \mathrm{~km}$ Northeast of Darwin, the NT's capital city, and has a population of 221 indigenous people living in 40 households,${ }^{57} 5.5$ per household. Ramingining and Gapuwiyak are both located in Northeast Arnhem Land, $450 \mathrm{~km}$ and $550 \mathrm{~km}$ from Darwin, respectively. Gapuwiyak is home to 871 indigenous residents in 101 households or 8.6 persons per household. Ramingining is the most crowded of the study communities with 811 indigenous residents in 92 households or 8.8 persons per household. ${ }^{44}$ 
Across these communities, for historical and geographical reasons, although English is spoken in all localities, there is a gradient in the degree to which traditional languages and conceptual constructs are retained and used in routine daily communication. One or more of the dialects of Yolngu matha are used for day-to-day communication in Gapuwiyak and Ramingining. In Minjilang, day-to-day communication is either in the local Kriol or in Gunwingku, the major language group in Western Arnhem Land. In Napranum, Torres Strait Kriol is typically used.$^{57}$ This diversity, among other factors, makes for cross-cultural challenges for developing educational and awareness-raising strategies that have meaning in terms of health behaviour for local community residents, particularly smoking. However, this diversity also provides the opportunity to examine the transferability of interventions across this cultural gradient.

\section{Overview of evaluation approach}

The study uses a mixed-methods exploratory evaluation design ${ }^{58}$ which is well suited for such community-focused interventions. These groups naturally form the evaluation advisory group for each community to be engaged and consulted throughout the project. First, the purposes of the project are discussed and explained in detail with this group and qualitative information from participant responses will provide guidance on intervention content and delivery, evaluation procedures and community requirements. Then, a rapid survey is to be conducted with as many heads of community households as possible to gauge their interest in having the project team visit their household to discuss the study objectives in more details (and in private). At each participating household, and where informed consent is provided, a first round of interviews with all senior household members will be conducted. In these interviews, the number of residents and resident smokers in age and gender groups will be documented and a household tobacco expenditure budget will be compiled. The nature and content of any existing household or individual strategies to control SHSe will be documented. The use of a particle metre to measure each household's SHSe will be discussed and negotiated and particle measures taken where permission is granted. Permission for a follow-up assessment and further particle measurements will be discussed and follow-up assessments will be conducted with those who agree. During the study period, tobacco sales data from ALPA's community store will be used to inform discussions about tobacco use and to monitor any abrupt changes which may occur in consumption or expenditure.

This study follows the National Health and Medical Research Council (NHMRC) guidelines on ethical research approaches to indigenous studies. The identified values that are integral to Aboriginal and Torres Strait Islander studies are spirit and integrity, reciprocity, equality, survival and protection and responsibility. ${ }^{59}$ These values are met with this project as it has been community inspired, includes an indigenous community advisory group and engages with communities in ways developed and managed by community members and their established service and advocacy groups.

\section{The 'Discovery Education' approach underpins the intervention}

The 'Discovery Education' model, the basis for the intervention, has been developed and used by ARDS over the past 30 years, but never systematically evaluated. ARDS contends that dialogue and understanding occur more rapidly and effectively in a people's first language and when founded in the shared conceptual structures in communities. ${ }^{59}$ To create effective dialogue on topics such as smoke-free homes, the methodology follows these conceptual steps (though in practice they can occur in parallel):

1. 'Discovery Education' commences by seeking to understand what people know of a topic and the questions they have about it. This enquiry identifies the differences between mainstream perspectives and those of community members.

2. The concepts and words in the vernacular that people apply to the subject at hand are identified. This methodological step aims to identify generative vocabulary, that is, keywords that relate to the topic.

3. Then, through further dialogue, new relevant knowledge is shared. 'Discovery Education' is a two-way process where both ARDS educators and indigenous community members learn about each other's worldview. During this process, new generative terms are found and improved ways of explaining new topics are developed.

The 'Discovery Education' process allows people to progressively integrate new knowledge into their existing worldview and to make their own decisions about how they will respond. Indigenous Australians have long been the subject of politically motivated 'interventions ${ }^{6061}$ and so welcome the two-way, democratic engagement between equals that is enshrined in the methodology. ${ }^{62}$

\section{Participants, recruitment, data and analysis 'Discovery Education' groups}

In each community, advised and guided by the research partners, and using existing networks and established relationships, 'Discovery Education' groups will be recruited comprised key community representatives. Purposive sampling will be combined with a snowball approach, where further recommendations for both participants to recruit and advice about recruitment processes will be sought. A sufficient number of focus group sessions will be conducted to represent the community's clan structure.

\section{Data}

The discussion thread for the 'Discovery Education' groups will generate qualitative information:

1. Shared information about why SHSe is a health issue and translation of the information into an appropriate form for local people. 
2. The utility of measurements of respirable particulate matter as an indicator of SHSe will be discussed while demonstrating the use of a particle measuring device (Dylos DC1700). The Dylos unit provides a cheap, real-time data source that can be used as a motivator to bring about change in smoking behaviours in homes ${ }^{19}$ particularly in homes where there are young children. ${ }^{16}$

3. Community people's awareness of health risks from SHSe and how they assess these.

4. How this information can best be conveyed to householders in the community as a whole.

5. Kinds of incentives that would encourage different groups in the community to implement SHSe strategies in their homes and how the incentives should be allocated. For example, our pilot data show that vouchers for purchases at the community store or to run household electricity consumption are welcomed. The 'Discovery Education' groups will assist in identifying culturally appropriate and effective rewards, along with the fairest way to distribute them.

\section{Data analysis}

'Discovery Education' group recordings will be transcribed and the text analysed thematically, moving from initial codes imposed by the discussion thread to descriptive and more analytic concepts. ${ }^{63} \mathrm{NVivo}$ V.10 will aid data retrieval and management for two researchers coding independently.

\section{Rapid survey}

Information about awareness of health risks of SHSe and how people assess these compiled in the 'Discovery Education' groups will be combined with evidence in the literature and used to develop culturally relevant instruments for survey data collection. The 'Discovery Education' groups will guide this process. The survey will be administered by interviewers, in local language at the ALPA-run community store whereas many adult indigenous community residents as possible will be provided information about the proposed community-led strategy and asked to participate in the survey.

\section{Data}

For those who consent, the brief survey will include assessments of:

- whether they are a smoker, any quit intentions or attempts in the past 12 months and whether they share their home with smokers;

- any current knowledge or awareness of SHSe and its harms;

- the location of the house they live in (lot number on the community services map) and whether they know of any household rules about smoking at that address;

- their interest or willingness to participate in or support the community-led strategy. Heads of households will be asked to identify as such through the rapid survey. Each self-reported head of household will be invited to participate in a more comprehensive survey to be conducted at their house and in private. Heads of households will be confirmed in consultation with members of the 'Discovery Education' group before proceeding.

\section{Data analysis}

Survey responses will be analysed using frequency distributions and cross tabulations of responses by lot number, managed and calculated in spreadsheets.

\section{Survey with heads of households}

\section{Baseline data}

The project aims to capture a minimum set of baseline data in each participating household.

- In consultation with the head of each household, demographic data, smoking status of residents and living conditions will be documented. A smoking grid, to record the smoking status of residents, for that day will be compiled using established methods. ${ }^{32} 64$

- For objective assessments of SHSe, households will be asked permission to set up the particle monitor inside the house temporarily. Self-reported assessments of SHSe will be elicited from heads of households and immediate feedback of particle monitoring results will be provided. ${ }^{1465}$

- Where permission is granted, SHSe will be measured over a 48 -hour period. ${ }^{58}$

- Any smoke-free spaces or rules about where people can or cannot smoke that are already established for that household will be described.

- How any smoke-free spaces and rules were originally established and how they are maintained will be documented.

- Whether household members would like to establish or expand smoke-free spaces and rules and receive incentives to do so will be discussed and documented.

- Weekly household expenditure on tobacco will be estimated.

Key outcome measure: the number of houses with rules about smoking in any household space assessed at baseline and followup

A home is considered smoke-free when no smoking is allowed indoors at all. ${ }^{66}$ Our preliminary data (unpublished) indicate that community residents similarly speak of SHSe controls in terms of smoking 'outside the house' which typically means that smoking is permitted on covered verandas and porches. Given the crowded housing these places can accommodate many people, particularly during the tropical wet season, ${ }^{6467}$ and many of these will be smokers. This potentially makes the delineation of a home as 'smoke-free' problematic and its enforcement very difficult. Additionally, our preliminary data indicate that smoke-free rules can be applied to separate rooms in a dwelling or 'around sick people or children', wherever they are located inside a house or on a veranda. Around one-third of household heads may 
have such rules in place, according to preliminary data for one study community. Their own interpretation may be that their houses are already 'smoke-free'. Given these complexities, our operational definition of a 'smoke-free home' will be:

'any home where there are any rules in place that are recognised by members of a household as constraining smoking in spaces indoors or on external verandas'.

In accordance with this definition, the status of 'smokefree homes' known in the community will be validated with the 'Discovery Education' groups both at baseline and follow-up using group discussions and consensus classification approaches already used routinely and shown to be valid in these populations to estimate tobacco use prevalence. ${ }^{49} 6869$ From 1 to 3 months after the baseline data collection, the objective assessments and the self-reported assessments will be repeated, following the steps specified above for the baseline data.

\section{Aims}

For a socially and environmentally significant change, more than $50 \%$ of the total number of participating houses in the community would come to have smokefree rules firmly in place at follow-up. This study aimed to bring about:

- an increase in the number of 'smoke-free' houses to greater than $50 \%$ of houses in the community

- a decrease in the mean SHSe levels observed in participating houses

- a decrease in household expenditure on tobacco which may also equate to a reduction in tobacco sales at the local community store.

\section{Data analysis}

At baseline, survey responses will be analysed using frequency distributions and cross tabulations of responses by lot number, calculated in spreadsheets. Descriptive statistics and graphical methods will be deployed as appropriate to the survey measures developed. Mainly categorical measures are anticipated, so $\chi^{2}$ tests and regression modelling will be used. Comparisons between baseline and follow-up will use McNemar's $\chi^{2}$ or Wilcoxon matched-pairs signed-rank tests for binary or categorical data, respectively. Paired t-tests will be used to compare baseline particulate matter and household tobacco expenditure with follow-up measurements. Weekly tobacco sales using data provided by ALPA will be used to provide an estimate per household expenditure and analysed using time series graphs of total weekly tobacco sales. These estimates will be compared with householder estimates. Quantitative data will be analysed using Stata V.14.

\section{Feedback of study results and consideration of feasibility} thresholds

In a final visit to each study community, 'Discovery Education' focus groups and advisory group members in the

$\begin{aligned} & \text { Table } 1 \\
& \text { time of writing }\end{aligned}$
\begin{tabular}{llllllllll}
\hline Community & 2017 \\
\hline 1 & FGs & RS & HS & F & & & & \\
\hline 2 & & & FGs & RS & HS & F & & \\
3 & & & & FGs & RS & HS & F & \\
4 & & & & FGs & RS & HS & F \\
\hline & & Completed & & & & & & \\
\hline
\end{tabular}

F, feedback result; FGs, focus groups; HS, household surveys; RS, rapid survey.

study advisory group will be reconvened to provide the study's results to clan leaders. Householders' self-assessments of SHSe at both baseline and follow-up that agree with objective SHSe measures would provide confidence in the qualitative information. A sound intervention study would enrol at least $80 \%$ of the houses in participating communities in a trial. A socially significant effect of the intervention would see an increase to at least $50 \%$ of households in participating study communities achieving validated 'smoke-free' status between baseline and follow-up. If this study can demonstrate a substantial proportion of households surveyed participating in the intervention and either succeeding or attempting to make changes, the case for expanding the trial would be compelling. Clan leaders will provide direction.

Timeline

The timeline for the study is as indicated in table 1 .

\section{DISCUSSION}

This project will reinforce the known capacity in remote indigenous Australian communities to manage environmental tobacco smoke ${ }^{67}$ and will encourage an increase in the number of household making concerted efforts to reduce SHSe in the study communities. Effective interventions to reduce SHSe in remote indigenous communities hold the prospect of reducing an array of significant smoking-related health issues. Such interventions for indigenous Australian populations have been advocated in the literature, ${ }^{686467}$ but none have been implemented or rigorously evaluated.

In a recent review of all the major community-level substance misuse intervention trials funded by Australia's NHMRC and conducted over the past decade in remote indigenous Australian communities, including five targeting tobacco, no clear effect in reducing smoking in these populations was found that could be attributed to any of the researcher-designed and evidence-based interventions. ${ }^{2}$ Low fidelity of intervention implementation, weak study designs, inadequate sample sizes and no explicit programme theory for intervention implementation and uptake were principal limitations described. ${ }^{2}$ Contrasting with these generally unsuccessful intervention designs, unique data from one NT study describe specific 
individual efforts by community residents to control SHSe in their dwellings. ${ }^{70}$ Pragmatic approaches which support such genuine community efforts are needed in these extreme circumstances of remoteness and crowded living circumstances where some of the highest smoking rates are known. There is a strong prospect that the efforts of community residents can be significantly strengthened as key indigenous stakeholders and community leaders in these regions also see smoke-free policies as a significant opportunity to reduce tobacco-related harms in their communities. $^{2}$

The ongoing strong community advocacy and support of the long-standing indigenous organisations leading this intervention (ALPA and ARDS) will carry on into the future representing an important opportunity to reduce SHSe and improve health outcomes in these remote communities. This meets the need for 'indigenous leadership, partnership and engagement and cultural tailoring' which has been strongly advocated in recent literature on the subject. ${ }^{71}$ With half or more of the people being daily smokers amidst the crowded living conditions found in these communities,${ }^{30}$ improvements to health experienced by other Australians as smoking rates fall have not been available in these marginalised populations. Future larger-scale interventions and more rigorous evaluations of their effectiveness will be possible if the intervention and evaluation approach being assessed here proves feasible with:

- robust self-reported and objective outcome measures

- evidence for strong implementation fidelity

- convincing potential for the community organisations initiating the intervention to sustain it with the transfer of positive intervention components becoming enabled.

\section{Limitations}

Notwithstanding the above-mentioned strengths of this study, several limitations must be acknowledged. Only a small number of communities could be included because of limited resources; the sample is as large as these resources have permitted. Additionally, it is an unavoidable weakness that there are no control communities included and no random selection of either communities or households. Random selection for such a pragmatic study is fraught with ethical difficulties in communities where the desire for assistance to make positive change is strong. The lack of randomisation cannot, however, be sustained as a criticism since the study communities were self-selecting, primarily because of the nature of the coalition between the study partners with a mandate to address the issue in the study communities. To keep faith with NHMRC guidelines which require maximum input from indigenous communities and agencies into research initiation and involvement in design and implementation, the study approach described here is arguably the only way such research can be conducted.

\section{Dissemination}

Strategies for disseminating study results will use the traditional means of scientific publications and professional conferences with a focus on reducing tobacco's harms. In addition, a targeted dissemination strategy will be used where messages will be tailored for specific audiences living and working within the study regions as designed for previous projects in this topic and setting. ${ }^{70}$ In-person presentations of results to the study advisory group in workshops is a feature of this approach. These will be facilitated by ARDS collaborators in the 'Discovery Education' cycle. For those with a mandate for policies in this topic area, 500-word policy briefs have been useful. ${ }^{70}$

Key practitioners in the study communities are indigenous Health Workers, resident Nurses and visiting Medical Officers. ${ }^{70}$ Although highlighting the need for clinical staff to support community-level initiatives, the heavy acute care and chronic disease treatment workload has made this very difficult for clinicians. ${ }^{72}$ Recognising this challenge, the study will develop from the evidence compiled a 'Smoke-free Space Practitioners Guide' aiming to foster smoke-free homes in other Aboriginal communities. As cross-cultural communication specialists, ARDS, ALPA and Apunipima can disseminate it through their established networks.

\section{CONCLUSIONS}

Very high and unchanging rates of smoking in populations where overcrowding in housing is extreme, but where there is documented potential ${ }^{6-8}{ }^{33-35}$ for appropriately designed and supported intervention strategies to be taken up by local indigenous community residents and advocated by their leaders, suggests that efforts to reduce SHSe will be welcomed and have high likelihood of success in these settings.

Acknowledgements The authors would like to thank the indigenous communities involved in the study and the study partners: Aboriginal Resource and Development Services (ARDS) Aboriginal Corporation, The Arnhem Land Progress Association, Apunipima Cape York Health Council, the Napranum Council and the Queensland and Northern Territory Heart Foundations. For reviewing the manuscript and contributing the map (figure 1), thanks to Mr Chris Rouen, Australian Institute of Tropical Medicine, James Cook University, Cairns.

Contributors ARC led in designing this study, involved in data collection, data analyses, drafting and approval of this manuscript. He designed the project and sought funding. KG involved with the Van Guard Grant since its approval and assisted with design, data collection, data analyses, drafting and approval of this study. JR involved with the Top-End smoke-free spaces project since 2012 and assisted in design, data collection, data analyses, drafting and approval of this study. MW is a consult for ARDS and is the organisational representative. MW involved in the design of the Arnhem Land project, and assisted in transferring the design of the study to the current location. MW also assisted in data collection, data analyses, drafting and approval of this study. NN is the organisational representative for Apunipima. NN was present during discussions for design and implementation of the study and assisted with data collection, data analyses, drafting and approval of this study. TF is the organisational representative for ALPA and assisted in data collection, data analyses, drafting and approval of this paper.

Funding This project is funded by the National Heart Foundation of Australia. Grant number 1014773.

Competing interests Although ALPA provides tobacco products for sale in the communities it supports these preventive efforts. Author TF is an ALPA employee 
who coordinate sales records including tobacco sales. TF will provide the tobacco sales data described in the Methods section.

Patient consent Obtained.

Ethics approval James Cook University Human Research Ethics Committee.

Provenance and peer review Not commissioned; externally peer reviewed.

Data sharing statement № additional unpublished data from this study protocol are available.

Open Access This is an Open Access article distributed in accordance with the Creative Commons Attribution Non Commercial (CC BY-NC 4.0) license, which permits others to distribute, remix, adapt, build upon this work non-commercially, and license their derivative works on different terms, provided the original work is properly cited and the use is non-commercial. See: http://creativecommons.org/ licenses/by-nc/4.0/

(C) Article author(s) (or their employer(s) unless otherwise stated in the text of the article) 2018. All rights reserved. No commercial use is permitted unless otherwise expressly granted.

\section{REFERENCES}

1. Oberg M, Jaakkola MS, Woodward A, et al. Worldwide burden of disease from exposure to second-hand smoke: a retrospective analysis of data from 192 countries. Lancet 2011;377:139-46.

2. Australian Government. Environmental tobacco smoke. Australia: Australian Government, 2014

3. Feleszko W, Ruszczyński M, Jaworska J, et al. Environmental tobacco smoke exposure and risk of allergic sensitisation in children: a systematic review and meta-analysis. Arch Dis Child 2014;99:985-92.

4. Centers for Disease Control and Prevention. Health Effects of Secondhand Smoke. 2017 https://www.cdc.gov/tobacco/data statistics/fact_sheets/secondhand_smoke/health_effects/.

5. Longman JM, Passey ME. Children, smoking households and exposure to second-hand smoke in the home in rural Australia: analysis of a national cross-sectional survey. BMJ Open 2013;3:e003128-8.

6. Thomas DP, Panaretto KS, Stevens M, et al. Smoke-free homes and workplaces of a national sample of Aboriginal and Torres Strait Islander people. Med J Aust 2015;202:S33-S8.

7. Edwards R, Gifford H, Waa A, et al. Beneficial impacts of a national smokefree environments law on an indigenous population: a multifaceted evaluation. Int J Equity Health 2009;8:12.

8. Thomas DP, Stevens M. Aboriginal and Torres Strait Islander smokefree homes, 2002 to 2008. Aust N Z J Public Health 2014;38:147-53.

9. Leeks KD, Hopkins DP, Soler RE, et al. Task Force on Community Preventive S. Worksite-based incentives and competitions to reduce tobacco use. A systematic review. Am J Prev Med 2010;38(2 Suppl):S263-74.

10. Morean ME, Camenga DR, Kong G, et al. Predictors of Middle School Students' Interest in Participating in an Incentive-Based Tobacco Prevention and Cessation Program in Connecticut. J Addict 2014;2014:1-6.

11. Glover M, Kira A, Walker N, et al. Using incentives to encourage smoking abstinence among pregnant indigenous women? A feasibility study. Matern Child Health J 2015;19:1393-9.

12. Kira A, Glover M, Walker N, et al. Recruiting Pregnant Indigenous Women Who Smoke into a High Contact Incentivized Cessation Trial: A Feasibility Study. Nicotine Tob Res 2016;18:2036-40.

13. Hikuroa E, Glover M. Reducing smoking among Indigenous Nursing Students using incentives/Te whakaheke I te kai paipa I waenga I nga tapuhi tangata whenua ma te whakamahi whakawhiwhinga. Nursing Praxis in New Zealand 2017;33:17-27.

14. Semple S, Apsley A, Azmina Ibrahim T, et al. Fine particulate matter concentrations in smoking households: just how much secondhand smoke do you breathe in if you live with a smoker who smokes indoors? Tob Control 2015;24(e3):e205-11.

15. Han I, Symanski E, Stock TH. Feasibility of Using Low-cost Portable Particle Monitors for Measurement of Fine and Coarse Particulate Matter in Urban Ambient Air. J Air Waste Manag Assoc 2016.

16. Klepeis NE, Hughes SC, Edwards RD, et al. Promoting smoke-free homes: a novel behavioral intervention using real-time audio-visual feedback on airborne particle levels. PLoS One 2013;8:e73251.

17. Liu Y, Beaucham CC, Pearce TA, et al. Assessment of two portable real-time particle monitors used in nanomaterial workplace exposure evaluations. PLoS One 2014;9:e105769.
18. Northcross AL, Edwards RJ, Johnson MA, et al. A low-cost particle counter as a realtime fine-particle mass monitor. Environ Sci Process Impacts 2013;15:433-9.

19. Semple S, Apsley A, Maccalman L. An inexpensive particle monitor for smoker behaviour modification in homes. Tob Control 2013;22:295-8

20. Zikova N, Hopke PK, Ferro AR. Evaluation of new low-cost particle monitors for PM2.5 concentrations measurements. J Aerosol Sci 2017:105:24-34.

21. Greenhalgh E, van der Sterren A, Knoche D, et al. 8.7 Morbidity and mortality caused by smoking among Aboriginal peoples and Torres Strait Islanders. Melbourne: Cancer Council Victoria, 2016.

22. van der Sterren A, Greenhalgh EM, Knoche D, et al. 8.3 Prevalence of tobacco use among Aboriginal and Torres Strait Islander people. Melbourne: Cancer Council Victoria, 2016.

23. Physicians for a Smoke-Free Canada. Smoking among Aboriginal Canadians. Australia: Physicians for a Smoke-Free Canada, 2013.

24. Greenhalgh E, Bayly M, Winstanley MH. International comparisons of prevalence of smoking. Melbourne: Cancer Council Victoria, 2015.

25. Ministry of health. Annual Update of Key Results 2015/16: New Zealand Health Survey. Australia: Ministry of Health, 2016.

26. MaW S. Tobacco in Australia: Facts and Issues: Melbourne Cancer Council Victoria. 2016 http://www.tobaccoinaustralia.org.au/chapter8-aptsi

27. Australian Government Department of Health. Tackling Indigenous Smoking (TIS) - National Statistics. 2017 http://www.health.gov.au/ internet/main/publishing.nsf/content/indigenous-tis-statistics

28. Australian Institute of Health and Welfare. Housing circumstances of Indigenous households: tenure and overcrowding. Canberra: Australian Institute of Health and Welfare, 2014.

29. Cancer Council. Tobacco in Australia: Facts and Issues. Melbourne: Cancer Council, 2011.

30. Campbell S, Bohanna I, Swinbourne A, et al. Stages of change, smoking behaviour and readiness to quit in a large sample of indigenous Australians living in eight remote north Queensland communities. Int J Environ Res Public Health 2013;10:1562-71.

31. Clough AR, Robertson JA, Maclaren DJ. The gap in tobacco use between remote Indigenous Australian communities and the Australian population can be closed. Tob Control 2009;18:335-6.

32. Clough AR, MacLaren DJ, Robertson JA, et al. Can we measure daily tobacco consumption in remote indigenous communities? Comparing self-reported tobacco consumption with communitylevel estimates in an Arnhem Land study. Drug Alcohol Rev 2011;30:166-72.

33. Johnston V, Thomas DP. What works in Indigenous tobacco control? The perceptions of remote Indigenous community members and health staff. Health Promot $J$ Austr 2010;21:45-50.

34. DiGiacomo M, Davidson PM, Abbott PA, et al. Smoking cessation in indigenous populations of Australia, New Zealand, Canada, and the United States: elements of effective interventions. Int J Environ Res Public Health 2011:8:388-410.

35. Cancer Council. Tobacco in Australia: Facts and Issues. Melbourne: Cancer Council, 2014.

36. Robertson J, Conigrave K, Ivers R, et al. Addressing high rates of smoking in remote Aboriginal communities--new evidence for GPs. Aust Fam Physician 2013;42:492-6.

37. Carol Watson JF, Alexander K. Service NTDoHaCIn: , ed. A survey of drug use patterns in Nothern Territory Aboriginal Communities. Northern Territory: Drug and Alcohol Bureau, 1988.

38. Borland R, Yong HH, Cummings KM, et al. Determinants and consequences of smoke-free homes: findings from the International Tobacco Control (ITC) Four Country Survey. Tob Control 2006;15(Suppl):iii42-50.

39. Australian Bureau of Statistics. Australian Aboriginal and Torres Strait Islander Health Survey: First Results, Australia, 2012-13. Canberra: Australian Bureau of Statistics, 2013.

40. Australian Government. 2013 National Drug Strategy Household Survey report. Australian: Australian Institute of Health and Welfare, 2014.

41. Australian Bureau of Statistics. National Aboriginal and Torres Strait Islander Social Survey, 2014-15. Canberra: Australian Bureau of Statistics, 2016.

42. Australian Bureau of Statistics. Census 2011: Aboriginal and Torres Strait Islander Population in Queensland. Canberra: Australian Bureau of Statistics, 2011.

43. Australian Bureau of Statistics. Remoteness Structure. Canberra: Australian Bureau of Statistics, 2014.

44. Australian Bureau of Statistics. Data By Region. Canberra: Australian Bureau of Statistics, 2017. 
45. Theo Vos BB, Stanly L, Lopez A. The burden of disease and injury in Aboriginal and Torres Strait Islander peoples 2003. Brisbane: The University of Queensland, 2007.

46. Vos T, Barker B, Begg S, et al. Burden of disease and injury in Aboriginal and Torres Strait Islander Peoples: the Indigenous health gap. Int J Epidemiol 2009;38:470-7.

47. Horton D. The Encyclopaedia of Aboriginal Australia: Aboriginal and Torres Strait Islander history, society and culture. Canberra: Aboriginal Studies Press, 1994.

48. Peterson N. Demand Sharing: Reciprocity and the Pressure for Generosity among Foragers. Am Anthropol 1993;95:860-74.

49. Clough AR, Guyula T, Yunupingu M, et al. Diversity of substance use in eastern Arnhem Land (Australia): patterns and recent changes. Drug Alcohol Rev 2002;21:349-56.

50. Robertson J. Top end tobacco project. The Chronical 2009;14.

51. Apunimpima. About. $2013 \mathrm{http}: / /$ www.apunipima.org.au/about

52. Apunimpima. Napranum. 2013 http://www.apunipima.org.au/ napranum

53. ARDS. Vision and Objectives. 2016 https://ards.com.au/about/visionobjectives/

54. ALPA. About ALPA. 2017 http://www.alpa.asn.au/pages/AboutALPA.html

55. Brimblecombe JK, McDonnell J, Barnes A, et al. Impact of income management on store sales in the Northern Territory. Med J Aust 2010;192:549-54.

56. Thomas DP, Ferguson M, Johnston V, et al. Impact and perceptions of tobacco tax increase in remote Australian Aboriginal communities. Nicotine Tob Res 2013;15:1099-106.

57. Australian Bureau of Statistics. 2016 Census QuickStats - Napranum. Canberra: Australian Bureau of Statistics, 2017.

58. Marsh J, McNeill A, Lewis S, et al. Protecting children from secondhand smoke: a mixed-methods feasibility study of a novel smoke-free home intervention. Pilot Feasibility Stud 2016;2:53.

59. National Health and Medical Research Council. Aboriginal and Torres Strait Islander Health Research Ethics A domestic and international review. Australia: Australian Institute of Aboriginal and Torres Straight Islander Studies, 2013.

60. Australian Government. Stronger Futures in the Northern Territory Act 2012. Australia: Australian Government, 2012.

61. Australian Human Rights Commission. Social Justice Report 2007. Australia: Australian Human Rights Commission, 2007.
62. ARDS Aboriginal Corporation. Staff Training Guide. 18. Australia: ARDS Aboriginal Corporation, 2016.

63. Braun V, Clarke V. Using thematic analysis in psychology. Qual Res Psychol 2006:3:77-101.

64. Stevenson LC, Bohanna I, Robertson JA, et al. Aboriginal people in remote communities in Arnhem Land (Northern Territory) restrict their smoking in some environments: Implications for developing and implementing interventions to reduce exposure to environmental tobacco smoke. Drug Alcohol Rev 2013;32:627-30.

65. Apelberg BJ, Hepp LM, Avila-Tang E, et al. Environmental monitoring of secondhand smoke exposure. Tob Control 2013;22:147-55.

66. Association RH. Smoke-free Housing for Property Managers \& Condo Associations Washington: Respiratory Health Association. 2017 http://www.lungchicago.org/smoke-free-housing-managers/

67. Robertson J, Pointing BS, Stevenson L, et al. "We made the rule, we have to stick to it": towards effective management of environmental tobacco smoke in remote Australian Aboriginal communities. Int J Environ Res Public Health 2013;10:4944-66.

68. Clough AR, Baille R, Burns CB, et al. Validity and utility of community health workers' estimation of kava use. Aust N Z J Public Health 2002;26:52-7.

69. Clough AR, D'Abbs P, Cairney S, et al. Emerging patterns of cannabis and other substance use in Aboriginal communities in Arnhem Land, Northern Territory: a study of two communities. Drug Alcohol Rev 2004;23:381-90.

70. Robertson JA, Conigrave KM, Ivers R, et al. Translation of tobacco policy into practice in disadvantaged and marginalized subpopulations: a study of challenges and opportunities in remote Australian Indigenous communities. Health Res Policy Syst 2012;10.23):23

71. Chamberlain C, Perlen S, Brennan S, et al. Evidence for a comprehensive approach to Aboriginal tobacco control to maintain the decline in smoking: an overview of reviews among Indigenous peoples. Syst Rev 2017;6:135.

72. Robertson J. Tackling tobacco: a call to arms for remote area nurses. Contemp Nurse 2010;37:49-56.

73. Australian Bureau of Statistics. Australian Statistical Geography Standard (ASGS): Volume 1 - Main Structure and Greater Capital City Statistical Areas. Canberra: Australian Bureau of Statistics, 2016. 